\title{
Glycoproteins Contained within Soamsan, a Traditional Oriental Medicine, are the Main Class of Active Ingredients Responsible for the Medicine-induced Immune Stimulation
}

\author{
Jeong-Chae Lee ${ }^{\dagger}$, Kyung-Yeol Lee ${ }^{\ddagger}$ Ha-Na Jung ${ }^{\ddagger}$ Jae-Gon Kim ${ }^{\S}$ and Yong-Suk Jang ${ }^{\ddagger *}$ \\ 'Department of Orthodontics and Institute of Oral Bioscience, Chonju 561-756, Korea \\ ${ }^{\star}$ Bank for Cytokine Research and The Institute for Molecular Biology and Genetics, Chonju 561-756, Korea \\ ${ }^{\S}$ Department of Pediatric Dentistry, Chonbuk National University, Chonju 561-756, Korea
}

Received 22 November 2004, Accepted 7 January 2005

\begin{abstract}
In our previous study, Soamsan, a traditional Oriental medicine, was shown to enhance the induction of antigenspecific immune responses, and it was speculated that the enhancing activity might be closely associated with glycoproteins contained within the medicine. To elucidate this speculation, protein samples from each component, used in the preparation of Soamsan, were obtained and their immune stimulating activities were tested with mouse splenocytes. All the samples markedly enhanced the lymphocyte proliferation and cytokine secretion by the mouse splenocytes. In particular, the enhancement was significantly higher with the protein sample treatments than with those of the original crude sample. Furthermore, the pronase $\mathrm{E}$ - and $\mathrm{NaIO}_{4}$-mediated inhibition of splenocytestimulation activity of the protein samples clearly supported that glycoproteins are the main class of active ingredients responsible for the lymphocyte stimulating activity of the samples. Consequently, our findings suggest that glycoproteins might have a pivotal role in Soamsanmediated immune modulation, although the in vivo effect of the glycoproteins should be further elucidated.
\end{abstract}

Keywords: Glycoproteins, Immune stimulation, Traditional medicine

\section{Introduction}

Among the traditional medicines, Soamsan has long been used to treat a variety of diseases and to maintain good health (Ha and Lee, 1998; Kim and Lim, 1999). This medicine is

*To whom correspondence should be addressed.

Tel: 82-63-270-3343; Fax: 82-63-270-4312

E-mail: yongsuk@chonbuk.ac.kr also thought to induce cancer remission and prevent chronic illnesses by modulating immune responses. In our previous study, we investigated whether Soamsan could act as an immune modulator using hen egg-white lysozyme as a model antigen system. For example, the oral administration of the medicine was demonstrated to potentially enhance antigenspecific antibody production as well as $\mathrm{T}$ cell-mediated immune responses (Kim et al., 2002b). In particular, the active ingredients responsible for the immune enhancing activity were tentatively speculated to be the glycoproteins contained within the medicine, although direct evidence for this speculation and the exact mechanism remain to be elucidated. However, this observation was in good agreement with the previous reports that the stimulatory activity of many medicinal herbs on lymphocyte proliferation, antibody production, and cytokine production are closely associated with glycoproteins (Yoshida et al., 1997; Shan et al., 1999; Lee and Lim, 2001).

In the present study, purified protein samples were prepared from water extracts of each component used in Soamsan, without a boiling process. The immune stimulating activity of the protein samples were determined using mouse splenocytes.

\section{Materials and Methods}

Chemicals, plastics and mice Unless otherwise specified, all chemicals and plastics used in this study were purchased from Sigma Chemical Co. (St. Louis, USA) and Falcon Labware (Becton-Dickinson, Franklin Lakes, USA), respectively. The cytokine reagents were obtained from BD PharMingen Inc. (San Diego, USA), and BALB/c mice (4-6 weeks old) were purchased from Damul Science (Yusung, Korea).

Preparation of protein samples All the herbal materials used to prepare the Soamsan were obtained from the College of Oriental 
Table 1. The yields from extracts of each Soamsan component $(50 \mathrm{~g})$

\begin{tabular}{lcc}
\hline \multirow{2}{*}{ Components } & \multicolumn{2}{c}{ Yield $(\mathrm{g})$ of extract $(\%$ of initial amount $)$} \\
\cline { 2 - 3 } & Original sample preparation & Protein sample preparation \\
\hline Angelicae gigantis Radix & $10.69(21.38)$ & $0.60(1.20)$ \\
Atractylodes macrocephalae Koidz & $4.69(9.38)$ & $0.32(0.64)$ \\
Ginseng Radix & $7.50(15.00)$ & $0.71(1.42)$ \\
Helianthus annuus L. & $2.87(5.74)$ & $0.42(0.84)$ \\
Laminaria japonica Aresch & $8.59(17.18)$ & - \\
Scutellaria barbata D. Don & $1.73(3.46)$ & $0.18(0.36)$ \\
Oldenlandia diffusa Roxb & $1.75(3.50)$ & $0.39(0.78)$ \\
Sargassum stenophyllum & $7.43(14.86)$ & $0.32(0.64)$ \\
Spargranii Rhizoma & $3.02(6.04)$ & $0.33(0.66)$ \\
Tribuli fructus & $4.03(8.06)$ & $0.18(0.36)$ \\
\hline
\end{tabular}

The samples were prepared from $50 \mathrm{~g}$ of each component used for Soamsan preparation as described in the Materials and Methods. There was no detectible protein sample harvested from the crude preparation of Laminaria japonica Aresch using the ammonium sulfate precipitation method.

Medicine, Wonkwang University (Iksan, Korea), and detailed in Table 1. The herbal plants assayed were authenticated by botanists at the Korea Institute of Oriental Medicine. Specimens of the plants were deposited in the herbarium of the College of Natural Sciences, Chonbuk National University (Chonju, Korea). Fifty grams of each dried component for the preparation of Soamsan were cut into small pieces, mixed with $500 \mathrm{ml}$ of distilled water in a screwcapped flask, and then shaken at $4^{\circ} \mathrm{C}$ for $3 \mathrm{~d}$. The resulting water extracts were filtered through filter paper (Whatman No. 3) and centrifuged at $5,000 \times g$ at $4^{\circ} \mathrm{C}$ for $10 \mathrm{~min}$. The collected supernatants were lyophilized to give dried powders of the original sample preparations. To prepare the protein sample preparations, the original preparations were dissolved with $80 \%\left(\mathrm{NH}_{4}\right)_{2} \mathrm{SO}_{4}$ to precipitate the proteins, and centrifuged to collect the precipitates. The precipitates were dialyzed against PBS and then lyophilized to yield a protein sample preparation. The yield of the sample preparations from each component of the medicine is shown in Table 1.

Cell cultures and treatment A single cell population of splenocytes was prepared and cultured using RPMI-1640 medium supplemented with antibiotics and 10\% FBS (HyClone, Logan, USA). One million cells per milliliter were resuspended in either 500 or $100 \mu \mathrm{l}$ of media for spreading onto either 24- or 96-well flatbottomed plates, respectively. Before the sample treatment, the culture media were replaced with fresh medium supplemented with only $0.5 \%$ FBS. At various times after the treatment, the cells were processed for analyses by trypan blue staining, tritium uptake, and ELISA assays.

Lymphocyte proliferation assay To assay the effect of the samples on the lymphocyte proliferation by immune cells, splenocytes, which had been cultured in 96-well flat-bottomed plates, were treated with varied concentrations of the samples (1$100 \mu \mathrm{g} / \mathrm{ml})$. Subsequently, $0.5 \mu \mathrm{Ci} / \mathrm{ml}$ of $\left[\right.$ methyl $\left.{ }^{3} \mathrm{H}\right] \quad \mathrm{TdR}$ (Amersham Pharmacia Biotech Inc., Piscataway, USA) was added to each well for the last $16 \mathrm{~h}$ of a $48 \mathrm{~h}$ culture period. The cells were harvested using a cell harvester (Inotech Inc., Switzerland) and the incorporated tritium contents were determined using a liquid scintillation counter (Packard Instrument Co.). In addition, the sample-mediated cytotoxicity was measured using a trypan blue exclusion experiment as previously described (Lee et al., 2003).

Measuring the cytokine levels The level of cytokines produced by the lymphocytes after the sample treatments were determined by ELISA, which was conducted by the Bank for Cytokine Research (Chonbuk National University, Chonju, Korea). Briefly, the splenocytes cultured in 24-well tissue culture plates were treated with different concentrations of the samples $(1-100 \mu \mathrm{g} / \mathrm{ml})$ for $48 \mathrm{~h}$. The culture supernatants were then collected, and the level of cytokines representing the stimulation of Th1-type (IFN- $\gamma$ and IL2), Th2-type (IL-4 and IL-5) T cells and macrophages (and/or B cells) (TNF- $\alpha$ ) was determined by ELISA. The amounts of cytokines produced were calculated from standard curves generated with recombinant cytokine proteins of known concentrations.

Characterization of active components In order to characterize the nature of the stimulating activity of the samples on splenocytes, the protein samples were treated with either pronase $\mathrm{E}$ or $\mathrm{NaIO}_{4}$ to degrade either proteins (Shan et al., 1999) or carbohydrate residues (Oka et al., 1987), respectively. Briefly, each protein sample (5 mg) preparations were incubated in $4 \mathrm{ml} 0.1 \mathrm{M}$ Tris- $\mathrm{HCl}$ buffer $(\mathrm{pH}$ 8.0), containing $0.6 \mathrm{mg}$ pronase $\mathrm{E}$ and $50 \mathrm{mM} \mathrm{CaCl}$ for $72 \mathrm{~h}$ at $30^{\circ} \mathrm{C}$. The reaction mixtures were then heated at $100^{\circ} \mathrm{C}$ for $10 \mathrm{~min}$ to inactivate the pronase $\mathrm{E}$, and then dialyzed against PBS. Alternatively, the protein samples $(5 \mathrm{mg}$ ) were incubated with 100 $\mu \mathrm{l} 0.1 \mathrm{M} \mathrm{NaIO}_{4}$ at $25^{\circ} \mathrm{C}$ for $4 \mathrm{~h}$, followed by the addition of $250 \mu \mathrm{l}$ $20 \%$ ethylene glycol. Finally, the samples were dialyzed against PBS.

To determine whether the protein samples contain lipopolysaccharide (LPS)-like compounds, the samples $(200 \mu \mathrm{g})$ were incubated for $2 \mathrm{~h}$ at $37^{\circ} \mathrm{C}$ in $500 \mu \mathrm{l}$ of the culture medium, containing 500 units of polymyxin B (PMB), before their addition to the splenocyte cultures, and tested to see if the PMB treatment could inhibit the proliferation mediated by LPS-like compounds.

Statistical analyses All the results are expressed as the mean \pm 
standard error (SE). A paired-sample $t$ test and a one-way ANOVA, using SPSS v. 10.0 software, were used to make statistical comparisons between the groups, with a $P$ value $<0.05$ considered statistically significant.

\section{Results and Discussion}

As a way to understand the mechanism of the soamsanmediated immune modulation, attempts were made in this study to identify the active ingredient(s) of the medicine. It has been reported that the stimulatory effects of many Oriental medicinal herbs on lymphocyte proliferation and cytokine production are associated with glycoproteins (Fang et al., 1985; Yoshida et al., 1997; Shan et al., 1999; Lee and Lim, 2001). Similar to these reports, our previous reports suggested that glycoproteins might act as immune modulators (Kim et al., 2002a; Kim et al., 2002b). In the present study, original and protein sample preparations were purified from each component used in Soamsan, but without a heating process, and the immune stimulating activity of the samples was examined.

As shown in Table 2, the treatments of all the original samples increased the tritium uptake by splenocytes. Especially, the original samples prepared from Atractylodes macrocephalae Koidz, Helianthus annuus L., Scutellaria barbata D. Don, and Oldenlandia diffusa Roxb, among ten components of the Soamsan, showed markedly increased stimulations of the splenocyte proliferation. Interestingly, the protein samples prepared from those four components of Soamsan showed significantly higher splenocytes stimulating activities than the control, and the activities were higher than those of the original samples for the same sample concentrations. This result suggested that the protein samples included the active ingredient(s) responsible for the stimulatory activity of the samples on the lymphocyte proliferation. None of the sample showed any cytotoxicity on the splenocytes in the cultures, at least at the concentrations tested in this study (data not shown).

The stimulatory effects of the samples on the lymphocytes were also examined by analyzing the cytokine production by the mouse splenocytes after the sample treatment (Tables 3). Table 3 shows that the original sample treatment stimulated the production of a higher level of IFN- $\gamma$ from the splenocytes than the control. As shown in the proliferation assay, the increase was significantly higher when the protein samples were treated than with the same concentration of the original samples. A similar pattern of increase was also observed in the IL-2 production when the samples were used to treat the splenocytes. Furthermore, the increase in TNF- $\alpha$ production from the splenocytes was also observed when the original samples were treated, with the increase being significantly greater when the protein samples were treated than with the original samples. However, there were no detectable changes in the levels of IL-4 and IL-5 after treating the splenocytes with either original or protein sample preparations (data not shown). These results suggested that the protein samples contained the active ingredient(s) responsible for the splenocyte-stimulating activity. In order to confirm this suggestion and tentatively characterize the ingredient(s), the protein samples were separated using SDS-PAGE, and the various molecular weight bands were clearly found with Schiff reagent staining (Fig. 1). To determine whether the splenocyte-stimulating activity of the protein samples was due to glycoproteins, the samples were treated with pronase $\mathrm{E}$, $\mathrm{NaIO}_{4}$, or $\mathrm{PMB}$, and then their abilities to stimulate

Table 2. Effects of the samples obtained from the components of Soamsan on the proliferation of mouse splenocytes

\begin{tabular}{lcc}
\hline \multicolumn{1}{c}{ Components } & \multicolumn{2}{c}{$\left[\right.$ methyl $\left.{ }^{3} \mathrm{H}\right]$-TdR uptake by spleen cells $(\mathrm{cpm})$} \\
\cline { 2 - 3 } & Original sample preparation & Protein sample preparation \\
\hline Angelicae gigantis Radix & $10,582 \pm 286$ & $14,286 \pm 1,422^{*}$ \\
Atractylodes macrocephalae Koidz & $14,889 \pm 378$ & $41,042 \pm 1,192^{* * *}$ \\
Ginseng Radix & $6,960 \pm 577$ & $6,584 \pm 272$ \\
Helianthus annuus L. & $27,254 \pm 572$ & $47,815 \pm 1,388^{* * *}$ \\
Laminaria japonica Aresch & $7,825 \pm 781$ & - \\
Scutellaria barbata D. Don & $19,740 \pm 358$ & $35,040 \pm 704^{* * *}$ \\
Oldenlandia diffusa Roxb & $19,778 \pm 991$ & $38,062 \pm 1,694^{* * *}$ \\
Sargassum stenophyllum & $8,977 \pm 1,183$ & $9,112 \pm 604$ \\
Spargranii rhizoma & $6,586 \pm 415$ & $7,931 \pm 665^{*}$ \\
Tribuli fructus & $11,116 \pm 879$ & $9,254 \pm 432^{*}$ \\
Control & $3,847 \pm 273$ & - \\
Concanavalin A (Con A) & $42,357 \pm 1,775$ & - \\
Lipopolysaccharide (LPS) & $44,298 \pm 2,472$ & - \\
\hline
\end{tabular}

BALB/c spleen cells $\left(1 \times 10^{6}\right.$ cells $\left./ \mathrm{ml}\right)$ were incubated with $10 \mu \mathrm{g} / \mathrm{ml}$ of the samples, Con A, or LPS for $48 \mathrm{~h}$ with $\left[\right.$ methyl $\left.{ }^{3} \mathrm{H}\right] \mathrm{TdR}$ incorporated for the last $16 \mathrm{~h}$. The results are expressed as the mean tritium uptake, in cpm $\pm \mathrm{SE}$ of triplicate experiments. $* p<0.05$ and $*_{* *} p<0.001$ indicate significant differences of the values between the original and protein sample preparations (paired-sample $t$ test). 
Table 3. Effects of the samples from the components of Soamsan on production of IFN- $\gamma$, IL-2, and TNF- $\alpha$ by mouse splenocytes

Sources of protein samples

IFN- $\gamma$ production $(\mathrm{ng} / \mathrm{ml}) \quad$ IL-2 production $(\mathrm{pg} / \mathrm{ml}) \quad$ TNF- $\alpha$ production $(\mathrm{ng} / \mathrm{ml})$

Original sample Protein sample Original sample Protein sample Original sample Protein sample

\begin{tabular}{lcccccc}
\hline Angelicae gigantis Radix & $2.10 \pm 0.18$ & $7.73 \pm 0.44 * * *$ & $2.12 \pm 0.77$ & $4.30 \pm 0.70^{*}$ & $3.27 \pm 0.99$ & $30.31 \pm 0.81 * * *$ \\
Atractylodes macrocephalae Koidz & $8.87 \pm 2.46$ & $45.12 \pm 6.24 * *$ & $1.98 \pm 0.88$ & $3.90 \pm 0.42^{*}$ & $7.76 \pm 2.30$ & $51.74 \pm 8.84 * *$ \\
Ginseng Radix & $4.21 \pm 0.13$ & $8.33 \pm 0.10^{* * *}$ & $2.1 \pm 0.75$ & $4.71 \pm 1.57$ & $3.32 \pm 1.20$ & $28.57 \pm 0.99 * * *$ \\
Helianthus annuus L. & $4.82 \pm 1.29$ & $11.42 \pm 2.16^{*}$ & $1.77 \pm 0.39$ & $3.65 \pm 0.65^{*}$ & $3.86 \pm 2.56$ & $28.98 \pm 4.99 * *$ \\
Laminaria japonica Aresch & $1.89 \pm 0.18$ & - & $1.49 \pm 0.78$ & - & $4.32 \pm 2.44$ & - \\
Scutellaria barbata D. Don & $2.43 \pm 0.12$ & $4.05 \pm 0.05 * * *$ & $1.56 \pm 0.41$ & $3.42 \pm 0.69 *$ & $4.83 \pm 2.45$ & $39.06 \pm 2.12 * * *$ \\
Oldenlandia diffusa Roxb & $4.79 \pm 0.21$ & $12.75 \pm 0.11 * * *$ & $1.87 \pm 0.50$ & $3.70 \pm 0.71^{*}$ & $6.27 \pm 1.29$ & $43.17 \pm 0.99 * * *$ \\
Sargassum stenophyllum & $4.21 \pm 1.04$ & $11.52 \pm 1.99 * *$ & $1.45 \pm 0.45$ & $1.34 \pm 0.84$ & $5.89 \pm 2.30$ & $34.57 \pm 7.15 * *$ \\
Spargranii Rhizoma & $5.57 \pm 2.76$ & $26.56 \pm 2.99 * *$ & $2.11 \pm 0.59$ & $7.41 \pm 2.01 *$ & $5.34 \pm 3.24$ & $34.69 \pm 4.01 * *$ \\
Tribuli fructus & $8.98 \pm 0.39$ & $26.48 \pm 0.68 * *$ & $1.89 \pm 0.67$ & $4.50 \pm 0.85^{*}$ & $4.66 \pm 2.61$ & $33.47 \pm 1.05 * * *$ \\
Control & $0.64 \pm 0.14$ & - & $1.40 \pm 0.72$ & - & ND & - \\
Concanavalin A (Con A) & $41.43 \pm 0.74$ & - & $4.377 \pm 402$ & - & $23.0 \pm 10.75$ & - \\
Lipopolysaccharide (LPS) & $43.86 \pm 3.78$ & - & $1.23 \pm 0.32$ & - & $51.74 \pm 10.91$
\end{tabular}

Splenocytes were cultured, and then exposed to $10 \mu \mathrm{g} / \mathrm{ml}$ of the original or protein samples for $48 \mathrm{~h}$. Con A and LPS were used as positive controls. The levels of IFN- $\gamma$, IL-2, and TNF- $\alpha$ production were analyzed by ELISA, and the results represent the mean \pm SE. ${ }^{*} p<0.05, * * p<0.01$, and $* * * p<0.001$ indicate significant differences of the values between the original and protein sample preparations (paired-sample $t$ test). The same experiments were repeated three times, and a representative result is shown in the table. ND represents the sample where the level was not determined.

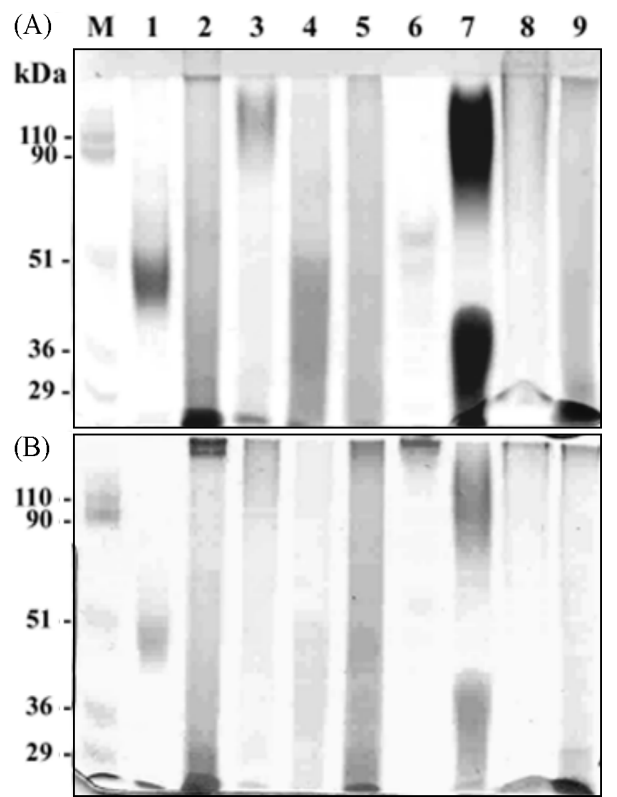

Fig. 1. SDS-PAGE analyses of protein sample preparations. The protein samples were loaded with $200 \mu \mathrm{g}$ per lane, electrophoresed in a $10 \%$ polyacrylamide mini-gel, and stained with CBB (A) or Schiff reagent (B). Lanes 1, 2, 3, 4, 5, 6, 7, 8, and 9 represent the samples from Ginseng Radix, Angelicae gigantis Radix, Scutellaria barbata D. Don, Tribuli fructus, Oldenlandia diffusa Roxb, Atractylodes macrocephalae Koidz, Spargranii Rhizoma, Sargassum stenophyllum, and Helianthus annuиs L, respectively. $\mathrm{M}$ represents a molecular weight marker.

lymphocyte proliferation and cytokine production were tested. As shown in Table 4, treating the samples with either pronase
$\mathrm{E}$ or $\mathrm{NaIO}_{4}$ apparently reduced the lymphocyte proliferative activity of the protein samples. The treatment also reduced the stimulating activity of the samples on the cytokine secretion by the mouse splenocytes (data not shown). Interestingly, however, PMB, an LPS inhibitor, did not reduce the stimulating activity of the samples (Table 4), although the LPS-mediated stimulation of the lymphocyte proliferation was clearly inhibited by the PMB treatment (data not shown). These results suggest that the protein samples contain major active ingredient(s) associated with the splenocyte-stimulating activity (Paik et al., 2003). Furthermore, as shown by the Schiff regent staining (Fig. 1) and the pronase E- and $\mathrm{NaIO}_{4^{-}}$ mediated inhibition (Table 4), the subsequent experiments clearly revealed that glycoproteins were the main class of active ingredient in the protein samples, and the protein samples were confirmed not to contain LPS-like compounds.

Typically, traditional medicines consist of 5 to 15 components and a single component contains a number of active ingredients associated with its medicinal efficacy (Chung et al., 2001). This means that quality control of the source materials is very important for the therapeutic effects of traditional medicines. In that sense, it could be speculated that glycoproteins could potentially be used as one of the indicators for confirming the quality of herbal plants, at least in the preparation of Soamsan. However, since it has been generally recognized that glycoproteins are digested and/or poorly absorbed at the gastrointestinal tract after oral intake, further detailed experiments should be performed to clearly understand the mechanism of the activity. The physicochemical property of the glycoproteins, and their effects in vivo, using the components to prepare Soamsan, are currently being 
Table 4. Effects of Pronase E, $\mathrm{NaIO}_{4}$, and PMB on the protein sample-induced stimulation of lymphocyte proliferation

\begin{tabular}{|c|c|c|c|c|}
\hline \multirow{2}{*}{ Sources of protein samples } & \multicolumn{4}{|c|}{$\left[\right.$ methyl $\left.-{ }^{3} \mathrm{H}\right]-\mathrm{TdR}$ uptake by spleen cells $(\mathrm{cpm})$} \\
\hline & Control & Pronase $\mathrm{E}$ & $\mathrm{NaIO}_{4}$ & PMB \\
\hline Angelicae gigantis Radix & $12,453( \pm 843)$ & $7,350( \pm 1,031)$ & $5,485^{*}( \pm 380)$ & $13,465( \pm 2,382)$ \\
\hline Atractylodes macrocephalae Koidz & $23,755( \pm 1,411)$ & $14,708 * *( \pm 864)$ & $8,024 * * *( \pm 894)$ & $20,456( \pm 1,433)$ \\
\hline Ginseng Radix & $8,634( \pm 492)$ & $5,720( \pm 1,237)$ & $5,188 *( \pm 296)$ & $8,808( \pm 786)$ \\
\hline Helianthus annuus L. & $28,887( \pm 772)$ & $17,574 * *( \pm 2,492)$ & $11,149 * * *( \pm 1,225)$ & $23,530( \pm 2,192)$ \\
\hline Scutellaria barbata D. Don & $21,105( \pm 852)$ & $11,627 * *( \pm 2,108)$ & $6,245 * * *( \pm 1,042)$ & $22,450( \pm 1,218)$ \\
\hline Oldenlandia diffusa Roxb & $21,345( \pm 707)$ & $13,077 *( \pm 1,807)$ & $7,290 * * *( \pm 624)$ & $21,022( \pm 2,280)$ \\
\hline Sargassum stenophyllum & $12,073( \pm 854)$ & $9,347( \pm 1,331)$ & $9,081( \pm 667)$ & $10,135( \pm 1,410)$ \\
\hline Spargranii Rhizoma & $7,569( \pm 458)$ & $6,638( \pm 746)$ & $8,549( \pm 731)$ & $6,089( \pm 662)$ \\
\hline Tribuli fructus & $9,702( \pm 621)$ & $7,342( \pm 414)$ & $6,015^{*}( \pm 525)$ & $10,358( \pm 1,216)$ \\
\hline
\end{tabular}

The protein samples were incubated in the presence of pronase $\mathrm{E}, \mathrm{NaIO}_{4}$, or $\mathrm{PMB}$ as described in the Materials and Methods. Ten micrograms per $\mathrm{ml}$ of each sample were added to the splenocytes to measure the proliferation induction activity, using the tritium incorporation assay. The results are expressed as the mean tritium uptake in cpm $\pm \mathrm{SE}$ of triplicate cultures. $* p<0.05$, $* * p<0.01$, and $* * * p<0.001$ indicate significant differences between the experimental and control values (one-way ANOVA).

characterized through further investigation. We are also determining whether other active ingredients responsible for the immune stimulation are presented in the $\left(\mathrm{NH}_{4}\right)_{2} \mathrm{SO}_{4}$ precipitates, because the lymphocyte proliferative activity of the protein sample was not completely reduced by pronase $\mathrm{E}$ or $\mathrm{NaIO}_{4}$ treatment.

Acknowledgments This study was supported by a grant, from the Korea Health 21 R\&D Project, the Ministry of Health \& Welfare, Republic of Korea (03-PJ1-PG3-220000001), to Dr. J.-C. Lee. The authors sincerely thank Dr. W.-H. Woo for providing the recipe and materials to prepare the Soamsan. Dr. Y.-S. Jang was supported by the Chonbuk National University for his sabbatical leave of absence at the University of Alabama at Birmingham to perform part of the experiments for this work. Dr. K.-Y. Lee were supported by the Chonbuk National University Post-Doctoral Fellowship Program.

\section{References}

Chung, S. H., Terashi, H., Rhodes, L. M., Moon, N., Dunham, W. R. and Marcelo, C. L. (2001) The effect of three Korean traditional medicines on the growth rate of cultured human keratinocytes. J. Ethnopharmacol. 74, 53-61.

Fang, J. N., Proksch, A. and Wagner, H. (1985) Immunologically active polysaccharides of Acanthopanax senticosus. Phytochemistry 24, 2619-2622.

Ha, J. Y. and Lee, H. (1998) Antitumor and immune modulatory effects of Gagamleejungtang in BALB/c mice. Kor. J. Oriental. Pathology 12, 73-81.

Kim, E. H., Lee, J. C., Kim, J., Son, Y. O., Jung, G. H. and Jang, Y. S. (2002a) Modulation of antigen-specific immune responses by the oral administration of a traditional medicine, Bo-yanghwan-o-tang. Immunopharmacol. Immunotoxicol. 24, 423-440.

Kim, E. H., Lee, J. C., Park, C. H., Lee, M. Y. and Jang, Y. S. (2002b) Soamsan, a traditional Korean medicine, enhances antigen-specific immune responses in low-responder mice via the combined activity of glycoproteins and endotoxins. Int. Immunopharmacol. 2, 1529-1540.

Kim, S. H. and Lim, J. S. (1999) Effects of Korean traditional medicine on murine hematopoiesis (I): Regulation of hematopoietic cytokine expression. Kor. J. Immunol. 21, 165174.

Lee, J. C., Kim, J. and Jang, Y. S. (2003) Ethanol-eluted extract of Rhus verniciflua Stokes inhibits cell growth and induces apoptosis in human lymphoma cells. J. Biochem. Mol. Biol. 36, 337-343.

Lee, J. C. and Lim, K. T. (2001) Inhibitory effects of the ethanol extract of Ulmus davidiana on apoptosis induced by glucoseglucose oxidase and cytokine production in cultured mouse primary immune cells. J. Biochem. Mol. Biol. 34, 463-471.

Oka, S., Shigeta, S., Ono, K. and Jyo, T. (1987) An epitope residing in carbohydrate chains of sea squirt antigen termed Girep. J. Allergy Clin. Immunol. 80, 57-63.

Paik, S. Y., Ra, K. S., Chang, I. S., Park, Y. C., Park, H. S., Baik, H. S., Yun, J. W. and Choi, J. W. (2003) Purification and characterization of complement-activating acidic polysaccharides from the fruits of Capsicum annuum. J. Biochem. Mol. Biol. 36, 230-236.

Shan, B. E., Yoshida, Y., Sugiura, T. and Yamashita, U. (1999) Stimulating activity of Chinese medicinal herbs on human lymphocytes in vitro. Int. J. Immunopharm. 21, 149-159.

Yoshida, Y., Wang, M. Q., Liu, J. N., Shan, B. N. and Yamashita, U. (1997) Immunomodulating activity of Chinese medicinal herbs and Oldenlandia diffusa in particular. Int. J. Immunopharmacol. 19, 359-370. 